\title{
Efficient Address Remapping in Distributed Shared-Memory Systems
}

\author{
LIXIN ZHANG \\ IBM Austin Research Lab \\ MIKE PARKER \\ Cray Inc. \\ and \\ JOHN CARTER \\ University of Utah
}

\begin{abstract}
As processor performance continues to improve at a rate much higher than DRAM and network performance, we are approaching a time when large-scale distributed shared memory systems will have remote memory latencies measured in tens of thousands of processor cycles. The Impulse memory system architecture adds an optional level of address indirection at the memory controller. Applications can use this level of indirection to control how data is accessed and cached and thereby improve cache and bus utilization and reduce the number of memory accesses required. Previous Impulse work focuses on uniprocessor systems and relies on software to flush processor caches when necessary to ensure data coherence. In this paper, we investigate an extension of Impulse to multiprocessor systems that extends the coherence protocol to maintain data coherence without requiring software-directed cache flushing. Specifically, the multiprocessor Impulse controller can gather/scatter data across the network while its coherence protocol guarantees that each gather request gets coherent data and each scatter request updates every coherent replica in the system. Our simulation results demonstrate that the proposed system can significantly outperform conventional systems, achieving an average speedup of $9 \mathrm{X}$ on four memory-bound benchmarks on a 32-processor system.
\end{abstract}

Categories and Subject Descriptors: C.0 [Computer Systems Organization]: General; C.4 [Computer Systems Organization]: Performance of Systems

General Terms: Design

Additional Key Words and Phrases: Smart memory controller, multiprocessors, address remapping, shadow address, cache coherence, performance evaluation

Authors' addresses: Lixin Zhang, IBM Austin Research Lab, MS 904/6C019, 11501 Burnet Road, Austin, TX 78758; email: zhangl@us.ibm.com; Mike Parker, 1050 Lowater Road, Chippewa Falls, WI 54729; email: map@cray.com; John Carter, 50 S. Central Campus Drive, Room 3190, University of Utah, Salt Lake City, UT 84112-9205; email: retrac@cs.utah.edu.

Permission to make digital or hard copies of part or all of this work for personal or classroom use is granted without fee provided that copies are not made or distributed for profit or direct commercial advantage and that copies show this notice on the first page or initial screen of a display along with the full citation. Copyrights for components of this work owned by others than ACM must be honored. Abstracting with credit is permitted. To copy otherwise, to republish, to post on servers, to redistribute to lists, or to use any component of this work in other works requires prior specific permission and/or a fee. Permissions may be requested from Publications Dept., ACM, Inc., 1515 Broadway, New York, NY 10036 USA, fax: +1 (212) 869-0481, or permissions@acm.org.

(C) 2006 ACM 1544-3566/06/0600-0209 $\$ 5.00$ 


\section{INTRODUCTION}

There is an increasing demand for scalable high-performance distributed shared-memory (DSM) systems from both government agencies and industrial users. Over the last two decades, microprocessor speeds have increased roughly $55 \%$ per year, while DRAM latencies have improved only $7 \%$ per year. Although these trends are showing signs of slowing, they are predicted to continue through at least the end of this decade [Moore 1965]. As a result of these trends, we are approaching a time when multiprocessor systems will have local memory latencies measured in thousands of processor cycles and remote memory latencies measured in tens of thousands of processor cycles. In addition, growing application dataset sizes are increasing pressure on memory systems and increasing demand for bisection bandwidth, which further limits the scalability of DSM systems.

Conventional DSM systems are built for applications with excellent data locality. They perform poorly on many important data-intensive applications that do not exhibit sufficient data locality. For instance, on a directory-based cache-coherent nonuniform memory access (CC-NUMA) machine- the predominant scalable DSM architecture - all data is managed at the granularity of a block. Memory blocks are typically the size of largest cache line, e.g., 32-256 bytes, to exploit spatial locality. Each block is associated with a fixed home node that tracks the global state of the block, e.g., its state (shared/exclusive) and the set of nodes that are caching a replica of the block. When a processor accesses data that is not in its local caches, it sends a message to the data's home node to request a copy. For applications with poor locality, such as the GUPS benchmark that performs random global word updates, this design is inefficient. Every time GUPS performs a word update, a CC-NUMA system must load a full block of data from the home node to local caches, update one word of this block, and then write the block back to the home node. Every word update suffers at least two round-trip network latency and consumes two full blocks of network bandwidth. When executing GUPS on a 32-processor Origin 3000 machine, the processors are idle more than $90 \%$ of the time, waiting for data to be loaded.

It is appalling that the sustained performance of existing DSM supercomputers on many applications is less than 5\% of peak performance [Graybill 2002]. This poor utilization is only expected to get worse over time due to growing memory-processor gap. The unavoidable conclusion is that the sustainable performance of future scalable DSM systems will be largely determined by their ability to reduce the number and/or overhead of both local and remote memory accesses.

As part of the Impulse project, we have demonstrated how adding an optional level of address translation at the memory controller (MC) can drastically reduce the number of local memory accesses required by data-intensive applications [Carter et al. 1999; Zhang et al. 2001]. Applications can configure the Impulse MC to respond to requests to shadow addresses, physical addresses that do not correspond to valid memory locations, by scattering/gathering data to/from local memory locations. Applications can use shadow addresses to create cache/TLB-friendly data structures, e.g., a column of an array laid out on 
row-major order can be mapped to contiguous shadow addresses before being loaded into the processor cache. In response to a cache line fetch of a shadow address, the Impulse MC fetches and compacts multiple sparse data items at disjoint memory locations into a dense cache line, which it returns to the processor.

An issue that arises when data can be accessed via multiple physical addresses is that of aliasing. Processor caches assume that data items are accessed via unique physical addresses, which may not be the case if data can be accessed via both a normal physical address and one or more shadow addresses. Our previous work has focused on single-node systems and relies on software to flush caches at appropriate times to ensure coherence between normal and shadow accesses.

In this paper, we examine an extension of Impulse to multiprocessor systems. In the proposed system, a shadow cache line can be composed of memory words ${ }^{1}$ from multiple nodes. We extend the coherence protocol to support cross-node word-grained coherent read/write operations.

In our multiprocessor Impulse design, the Impulse controller on the node where a shadow request is made translates it into a series of real physical addresses, which may belong to either local or remote memory. The Impulse controller then sends a fine-grained GET (for load) or PUT (for write) request to the home node of each of these real physical addresses. When the home node receives a GET request, it loads the coherent value of the target word from local memory or a processor cache, depending on the state of the block containing the word. When the home node receives a PUT request, it sends a word-update request to local memory and every processor cache that has a copy of the block containing the target word. Data gathered in the shadow space is not automatically kept coherent with its original source, similar to how the Cray T3E [Scott 1996] treats data loaded into E-registers or how conventional CPUs treat data loaded into processor registers. In Section 3.2 we show that this model is not only easy to implement but also applicable to a majority of HPC applications.

Gathering/scattering data in multiprocessors yields several performance benefits. Like on a uniprocessor, gathering sparse data into dense cache lines improves spatial locality. This improved spatial locality can significantly improve cache and TLB hit rates, thereby reducing the number of local and remote memory accesses performed. By not loading unused data into caches, cache pollution and system bus bandwidth requirements are also reduced. Loading and storing sparse data at a fine granularity reduces network bandwidth requirements and memory pressure. Our simulation results show that the proposed system speeds up four data-intensive benchmarks by factors of $4.9 \mathrm{X}$ to $24.4 \mathrm{X}$, with a geometric mean speedup of $9 \mathrm{X}$ on a 32-processor system.

The remainder of this paper is organized as follows. Section 2 gives an overview of the Impulse architecture, including the basic idea, hardware organization, and remapping mechanisms. Section 3 describes the extensions that we propose to make scatter/gather work in DSM systems. Section 4 presents the

\footnotetext{
${ }^{1}$ In this paper, we use the term word to refer to data items smaller than or equal to a block. A word can be as small as four bytes or as large as a block.
} 


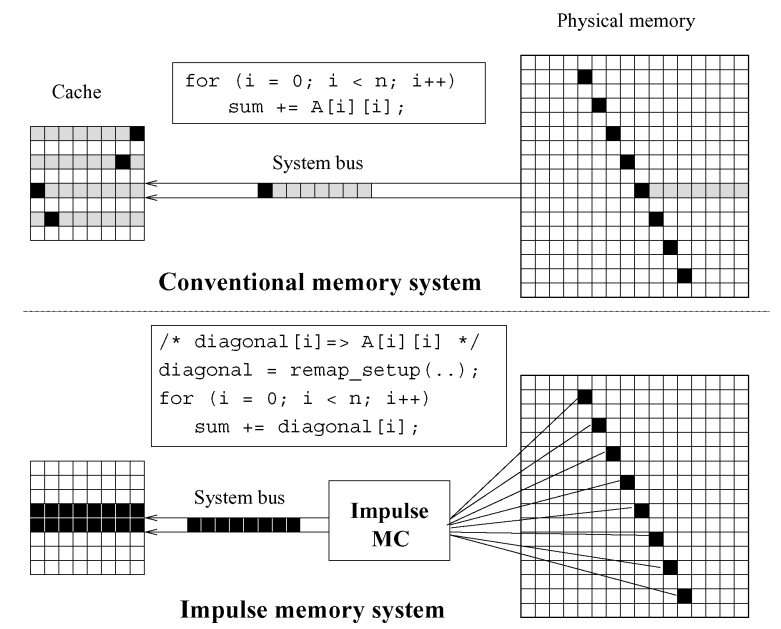

Fig. 1. Using Impulse to remap the diagonal of a dense matrix into dense cache lines. The black boxes represent elements on the diagonal, whereas the other boxes represent nondiagonal elements.

experimental results. Section 5 describes related work. Section 6 summarizes our conclusions and describes future work.

\section{OVERVIEW OF IMPULSE}

Impulse expands the traditional virtual memory hierarchy by adding address translation hardware to the memory controller. This optional extra level of remapping is enabled by the fact that not all physical addresses in a traditional virtual memory system typically map to valid memory locations. In Impulse terminology, the unused physical addresses constitute a shadow address space. Software configures the Impulse memory controller to interpret shadow addresses according to the application's need. Data items whose physical DRAM addresses are not contiguous can be mapped to contiguous shadow addresses. Virtualizing unused physical addresses in this way can improve the efficiency of on-chip caches and TLBs.

The operating system manages all resources in the expanded memory hierarchy and provides an interface for applications to specify optimizations for particular data structures. To exploit Impulse, system calls must be inserted into the application code to configure the memory controller. In response to an Impulse system call, the OS allocates a range of contiguous virtual addresses large enough to map the elements of the new (synthetic) data structure. The OS then maps the new data structure through shadow memory to the corresponding physical data elements.

As an example of how Impulse's remapping can be used, consider a program that accesses the diagonal elements of a large, dense matrix A. The physical layout of part of the matrix A is shown on the right-hand side of Figure 1. On a conventional memory system, each time the processor accesses a new diagonal element (A [i] [i]), it requests a full cache line of contiguous physical memory and accesses only a single word of each of these cache lines. Such an access is shown in the top half of Figure 1. 


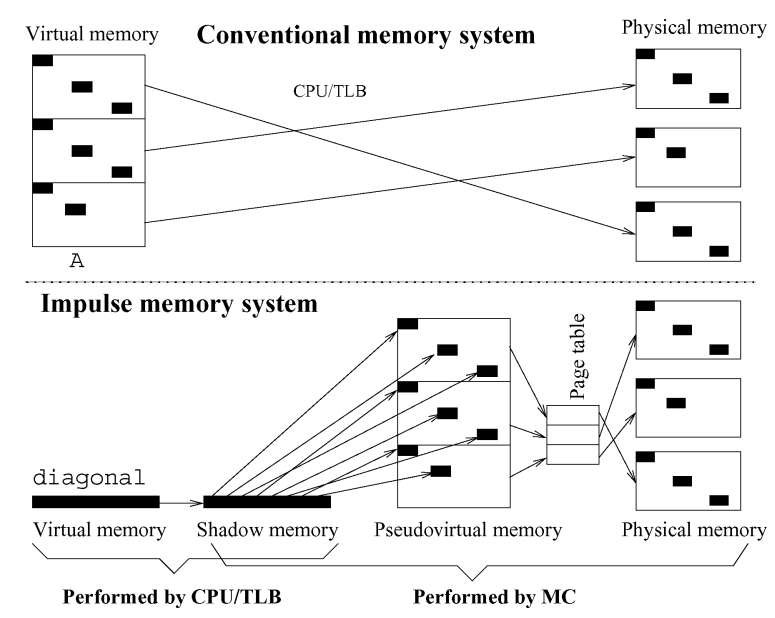

Fig. 2. Accessing the (sparse) diagonal elements via a dense diagonal vector.

To use Impulse, the application first calls remap_setup() to create a dense alias vector in the shadow space, diagonal, which contains just the diagonal elements. After remapping, the application accesses the diagonal elements via the new alias vector instead of the original matrix, i.e., using diagonal [i] in the place of A [i] [i]. Such an access is shown in the bottom half of Figure 1.

Upon seeing an access to a shadow address in the synthetic diagonal data structure, the memory controller needs to gather the corresponding diagonal elements from the original array, pack them into a dense cache line, and return this cache line to the processor. To determine where these sparse data items reside in physical memory, the MC first recovers their offsets within the original data structure, so-called pseudovirtual addresses. It then translates these pseudovirtual addresses to physical DRAM addresses using a page table. Figure 2 depicts the address translations for both the original matrix and the remapped image of its diagonal. As this figure shows, the pseudovirtual address space page layout mirrors the virtual address space, allowing Impulse to remap data structures that lie across noncontiguous physical pages. The shadow $\rightarrow$ pseudovirtual $\rightarrow$ physical mappings all take place within the memory controller.

\subsection{Hardware Organization}

Figure 3 shows the hardware organization of the Impulse memory controller. The core component is the shadow engine. The shadow engine contains a small SRAM (Assembly Buffer) to be the staging area for scattering/gathering data, shadow registers to store remapping configuration information, an ALU unit (AddrCalc) to translate shadow addresses to pseudovirtual addresses, and a Memory Controller Translation Lookaside Buffer (MTLB) to cache recently used translations from pseudovirtual addresses to physical addresses.

The extra level of address translation in the MC is optional. Valid physical addresses are passed untranslated to the DRAM interface (a). Shadow addresses must be converted to physical addresses before being presented to the DRAM 


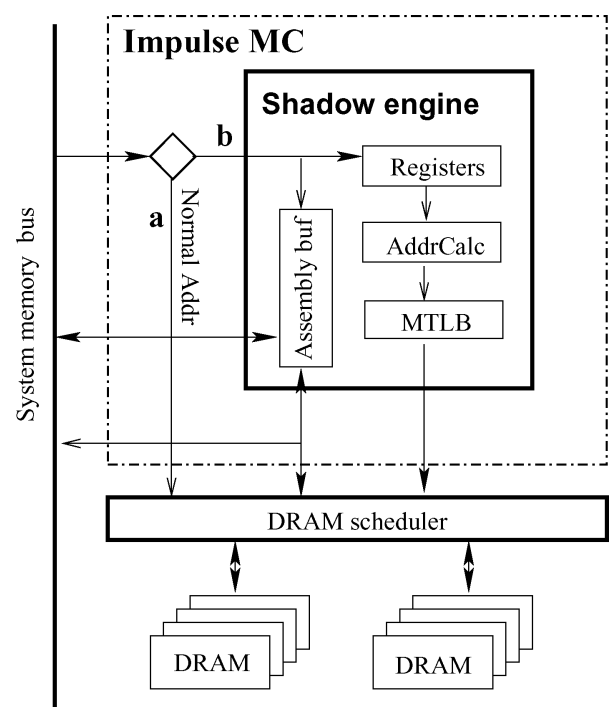

Fig. 3. Impulse memory controller.

by the shadow engine (b). To do so, the shadow engine first determines which shadow register set to use and passes its contents to the AddrCalc unit. The output of the AddrCalc is a series of pseudovirtual addresses for the individual sparse elements that need to be fetched. These pseudovirtual addresses are passed through the MTLB to be translated into physical addresses. The MTLB may generate exceptions. How to cope with such exceptions is discussed in an earlier publication [Swanson et al. 1998].

We have implemented a synthesizable Verilog version of the uniprocessor Impulse memory controller. The ASIC version has about one million transistors (including approximately $100 \mathrm{Kbits}$ of dual-ported SRAM), 650 pins, and a die size of about $80 \mathrm{~mm}^{2}$.

\subsection{Remappings}

Shadow address translations can take three forms: direct, strided, or indirect. A direct mapping is a coarse-grain translation that maps a shadow cache line directly to a contiguous line of physical memory. This mapping can be used to recolor physical pages without copying or to construct superpages dynamically [Swanson et al. 1998; Fang et al. 2001]. A strided mapping creates dense cache lines from strided array elements and can be used to optimize applications with nonunit strided accesses, e.g., column-major accesses to matrices stored in row-major order. Indirect mapping uses an indirection vector vec to translate an address soffset in the shadow space to pseudovirtual address vec[soffset]. A variation of this mapping allows the processor to send a cache line worth of addresses to the MC, asking the MC to gather a cache line of data based on these addresses [Zhang et al. 2000]. This mapping can optimize applications like SMVP (sparse matrix-vector product) and GUPS by replacing indirect 
accesses $(A[i d x[i]])$ or random accesses $(A[$ random(i) $])$ with sequential accesses (alias_A [i]).

For this study, we consider only strided and indirect mappings. Direct mappings do not require data to be gathered from multiple sources, so they can be handled as a trivial subcase of the fine-grained remappings.

\subsection{Memory-Side Prefetching}

Gathering a cache line in shadow memory can be much more expensive than fetching a single dense cache line depending on how the individual words being gathered are distributed across DRAM chips/banks. However, a single gathered cache line fill replaces multiple full cache line fills for the lines containing the gathered data, so the number of cache and TLB misses is reduced dramatically. The benefit of eliminating cache and TLB misses typically far outstrips the negative impact of the increased memory latency to load the gathered cache line. In addition, the Impulse MC uses prefetching to hide some of the gather/scatter latency. In particular, the shadow engine can perform sequential prefetching in the shadow address space. Prefetched (pregathered) shadow cache lines are stored in the assembly buffer. A hit in the assembly buffer returns a cache line to the processor immediately without going through a full address translation and data gathering process, as well as initiating the prefetch of the next line.

\subsection{Compiler Automation}

Requiring programmers to manually exploit Impulse's remapping features introduces a significant programming burden. To alleviate this burden, a compiler that can automatically apply remapping to applications has been developed, implemented, and evaluated [Huang et al. 2001]. The compiler uses locality analysis to determine if certain array references will benefit from remapping. It then uses a cost model to estimate the cost of setting up remappings, the cost of caches misses before and after remapping, and the number of cache misses before and after remapping. If the cost model shows that a remapping gives a $5 \%$ or better speedup, the remapping will be applied.

\section{MULTIPROCESSOR EXTENSIONS OF IMPULSE}

We call the multiprocessor version of Impulse Impulse-MP. Its hardware organization is presented in Section 3.1 and its coherence protocol extensions are described in Section 3.2.

\subsection{Hardware Organization}

Figure 4 presents the high-level view of a node in a Impulse-enabled DSM system. A node consists of one or more processors, each with private on-chip caches. Processors are connected to a Hub chip that incorporates a crossbar that connects to the network interconnect, local memory/directory controller, local I/O, and the shadow engine.

Unlike in uniprocessor systems, the shadow engine no longer resides in the memory controller. Instead, it is now an independent unit connected to the crossbar. An address issued to the Hub from a local processor can be a normal 


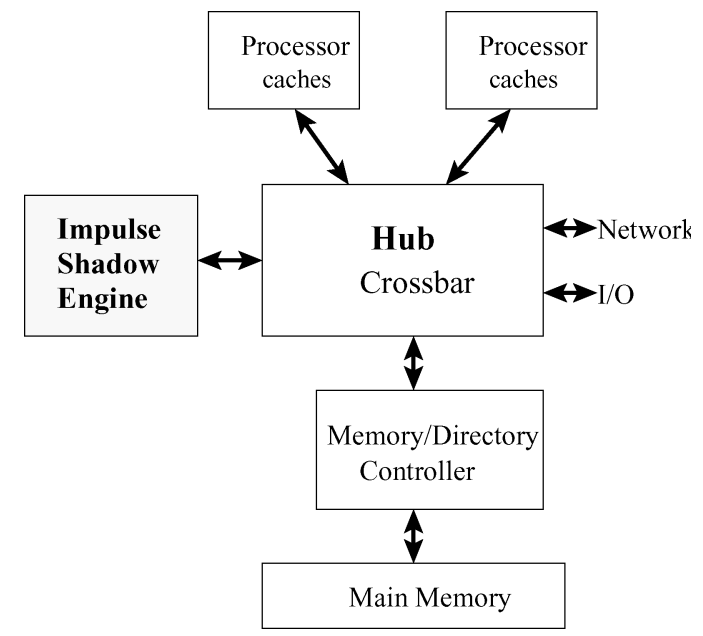

Fig. 4. Node overview.

physical address or a shadow address. If it is a normal address, it is sent to the local memory/directory controller (if it is a local memory access) or the network interface (if it is a remote access). If it is a shadow address, it is always routed to the local shadow engine. The shadow engine translates shadow requests into a series of real physical addresses and sends word-grained requests via the interconnect to the home nodes for each required word.

\subsection{Coherence Protocol Extensions}

Remapping may cause a data item to be cached through a physical address and one or more shadow addresses at the same time (so-called aliasing). Aliasing introduces two challenges: (i) ensuring that gather requests load coherent data and that scatter requests update all replicas of the modified data and (ii) keeping gathered data coherent after it is cached, even if the underlying data is modified via a different address. Uniprocessor Impulse relies on software to flush caches at the appropriate time to avoid aliasing. While it is easy to detect when and where cache flushing is needed to ensure coherency in serial programs, it is rather difficult to do so in parallel programs where more than one processes may be accessing the same data simultaneously via different addresses. As such, in Impulse-MP we provide additional hardware support to automate coherence handling for the most important cases.

To maintain full coherence, when the $\mathrm{MC}$ receives a shadow address, it must find all real physical addresses that the shadow address maps to; when the MC receives a normal address, it must find all shadow addresses to which the normal address is related. While shadow $\rightarrow$ physical translation is already done by the shadow engine, physical $\rightarrow$ shadow translation would require a similar unit with an inverted page table for every physical page. However, our investigation done with our industrial partner reveals that such a unit is unnecessary for the set of applications Impulse-MP targets, as those applications tend to 
access data in one particular way at a time. A main goal of Impulse-MP is to run vector applications efficiently on conventional general-purpose microprocessors. Vector machines like the Cray XMP [Cheung and Smith 1986] and Cray T3E [Scott 1996] can load data to vector registers directly from memory, without breaking data coherency or consistency. Similarly, Impulse-MP handles gather/scatter operations as vector register loads/stores, without resorting to more complicated coherence mechanisms or hardware units. Thus, we propose a simpler mechanism that guarantees each gather operation gets coherent data and each scatter operation correctly updates coherent copies in the entire system, but does not keep data coherent while it is cached in gathered form if the underlying data is modified via a different address.

We augment the coherence protocol to support word-grained GET/PUT operations. A fine-grained GET request loads a coherent value of a word. When the directory controller receives a GET request, it loads the latest value of the target word either from local memory or a processor cache, depending on the state of the cache line containing the word. However, it neither changes the state of the corresponding cache line nor adds the requesting processor into the list of sharers. A fine-grained PUT request updates a single word of a cache line. When the directory controller receives a PUT, it sends an update request to local memory and every processor that has a copy of the cache line containing the word updated. As with GET, the directory controller does not change the state of the corresponding cache line. In essence, the GET/PUT mechanism implements a release-consistent word-grained write update protocol. A word read by a GET operation is not kept coherent with other copies in the system and can be updated locally, while a subsequent PUT operation of the word acts as a release point to update other copies.

However, GET/PUT in the presence of remapping might cause problems for applications that have read-write or write-write conflicts to the same data at different addresses. In these cases, the compiler or programmer should replace normal accesses with corresponding shadow accesses. If normal accesses cannot be replaced by shadow accesses, e.g., because of the difficulty of figuring out the right shadow addresses, remapping should not be performed. In our experience, these conflicts occur rarely because (i) data is typically accessed via a single set of addresses at a time (e.g., via normal physical addresses during row-major walks and shadow addresses during column-major walks) or (ii) concurrent accesses to the same data structure via different addresses are all reads. The same compiler techniques used in vector machines can be used to detect such conflicts and generate correct code.

Sending an update involves transferring data to a remote cache. Microprocessors typically do not allow external devices to "push" data into their caches. To overcome this limitation, we propose to use a Remote Access Cache (RAC), such as employed by the DASH [Lenoski et al. 1990] and STiNG [Lovett and Clapp 1996] architectures. A conventional RAC stores remote data that is forced out of local processor caches, which can reduce the number of remote misses induced by limited processor cache capacity. With an RAC, when an update message is received by a hub, the hub sends an intervention request to the processor cache to force the line to be flushed. The flushed line is then placed in the RAC and the 
Table I. Configuration of the Simulated Based Systems

\begin{tabular}{|l|l|}
\hline Parameter & \multicolumn{1}{|c|}{ Value } \\
\hline Processor & 2 GHZ, four-way issue, 48-entry active list \\
\hline TLB & 128 entries, 16 K base pages \\
\hline L1 I-cache & Two-way, 32 KB, 64-byte lines, 1-cycle latency \\
\hline L1 D-cache & Two-way, 32 KB, 32-byte lines, 2-cycle latency \\
\hline L2 cache & Four-way, 2 MB, 128-byte lines, 10-cycle latency \\
\hline System bus & 8B CPU to system, 16B system to CPU \\
\hline & maximum 16 outstanding cache misses \\
\hline DRAM & 800 MHZ, 16 20-bit DDR channels, 100 processor cycles \\
\hline Network hop & 50 processor cycles \\
\hline Base Page Size & $16 \mathrm{~KB}$ \\
\hline
\end{tabular}

update is applied to the line in the RAC. When the processor next accesses the data, the access will be satisfied from the RAC, which is a much lower latency operation than a remote memory access.

\section{PERFORMANCE EVALUATION}

\subsection{Simulation Environment}

We use the execution-driven simulator UVSIM in our performance study. UVSIM is derived from the Impulse simulator URSIM [Zhang 2000]. It models a future-generation SGI DSM supercomputer, including a directory-based SN2MIPS-like coherence protocol [SGI 2001] that supports fine-grained updates as describe in Section 3.2. Each simulated node contains two MIPS R18000like [MIPS 2002] microprocessors connected to sysTF busses [SGI 2002]. Also connected to the bus is SGI's future-generation Hub [SGI 2002], which contains the processor interface, memory controller, directory controller, network interface, IO interface, and the proposed shadow engine.

Table I lists the major parameters of the simulated systems. The L1 cache is virtually indexed and physically tagged. The L2 cache is physically indexed and physically tagged. The DRAM backend has 16 20-bit channels connected to DDR DRAMs, which enables us to read an 80-bit burst every two cycles. Of each 80 -bit burst, 64 bits are data. The remaining 16 bits are a mix of ECC bits and partial directory state. DRAM chip latency is 100 processor cycles. The system bus and memory controller add another 36 cycles to the total memory access latency.

The simulated interconnect subsystem is based on SGI's NUMAlink-4. The interconnect is built using 16-port routers in a fat-tree structure, where each nonleaf router has eight children. We model a network hop latency of $25 \mathrm{nsec}$ (50 processor cycles). The minimum network packet is 32 bytes.

We have validated the core of our simulator against an SGI Origin 3000 system by setting the simulator's configurable parameters to match those of the real system. We compared the performance characteristics, such as total run-time and cache miss rates, by measuring a large mix of benchmarks on both the simulator and the real system. The simulator-generated results are all within $20 \%$ of the corresponding numbers generated by the real machine, and most are within $5 \%$. 
Table II. Benchmark Inputs

\begin{tabular}{|l|l|}
\hline Benchmark & \multicolumn{1}{|c|}{ Input } \\
\hline GUPS & $256 \mathrm{MB}$ table, $2 \mathrm{M}$ accesses \\
\hline Matrix & $640 \mathrm{~K} \times 640 \mathrm{~K}$ sparse matrix, 6M non-zeros \\
\hline Transitive & $4096 \times 4096$ integer matrices \\
\hline CornerTurn & $4096 \times 4096$ double matrices \\
\hline
\end{tabular}

\subsection{Benchmarks}

To evaluate the performance of Impulse-MP, we examine four benchmarks: GUPS, Matrix, Transitive, and CornerTurn from the DIS (Data-Intensive Systems) Stressmarks suite [AAE Corp. 2000]. We do not use the other four stressmarks because (i) Pointer and Update have the same access pattern as GUPS and (ii) Neighborhood and Field perform regular accesses and have excellent $\mathrm{TLB} /$ cache hit rates on conventional systems.

The synthetic GUPS benchmark performs random global updates and is the principal benchmark of interest for the National Security Agency. We optimize GUPS by precomputing a cache line's worth of addresses and then using indirect remapping to gather two cache line's worth of double precision values using these addresses.

The Matrix benchmark solves the linear equation $A \times p=b$, where $A$ is a sparse matrix, using the conjugate gradient method. Its core loop implements an SMVP (sparse-matrix vector product) operation. We use indirect remapping to replace accesses to the sparse matrix with sequential accesses to the shadow space.

The Transitive benchmark is an implementation of the Floyd-Warshall allpairs shortest path algorithm [Cormen et al. 1990]. It accepts an adjacency matrix representation of a directed graph and uses a recurrence relationship to produce the adjacency matrix of the shortest-path transitive closure. Its kernel is a three-level perfectly nested loop that accesses two matrices in column-major order while they are stored in row-major order. We change the column-major accesses to row-major accesses via transpose remapping, a variation of stride remapping that creates the shadow image of the transpose of a matrix.

The CornerTurn benchmark transposes a two-dimensional matrix using copying. In the original version of this benchmark, the source matrix is accessed in column-major order and the destination matrix is accessed in rowmajor order. We employ transpose remapping on the source matrix to replace its column-major with row-major accesses.

UVSIM directly executes statically linked 64-bit MIPS-IV application executables and supports the OpenMP runtime environment. All benchmark programs used in this paper are OpenMP-based parallel programs.

\subsection{Results}

Table II gives the input size of each benchmark. These input sizes are relatively small compared to real workloads. We choose them because they can be simulated in a reasonable amount of time, yet are large enough to put realistic pressure on the memory/network system and demonstrate the effectiveness 


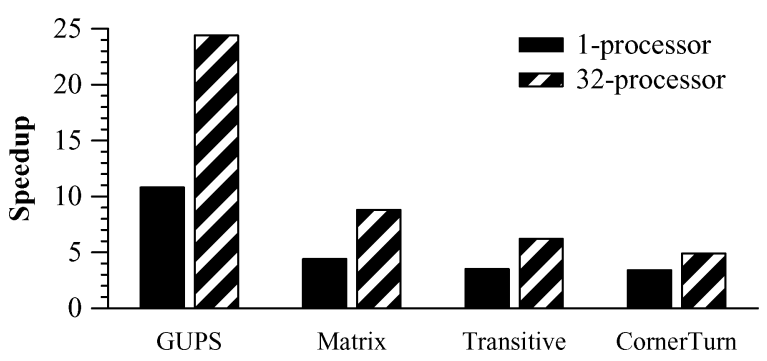

Fig. 5. Speedups of Impulse-MP.

Table III. TLB Misses and Memory Accesses and Latencies

\begin{tabular}{|c|c|c|c|c|c|c|}
\hline \multirow[b]{2}{*}{ Benchmark } & \multicolumn{2}{|c|}{ TLB Misses } & \multicolumn{2}{|c|}{ Local/remote Accesses } & \multicolumn{2}{|c|}{ Local/remote Latency } \\
\hline & Base & Remap & Base & Remap & Base & Remap \\
\hline GUPS & $2.25 \mathrm{M}$ & $17 \mathrm{~K}$ & $0.19 \mathrm{M} / 2.33 \mathrm{M}$ & $0.04 \mathrm{M} / 0.13 \mathrm{M}$ & $220 / 478$ & $128 / 679$ \\
\hline Matrix & $4.48 \mathrm{M}$ & $8 \mathrm{~K}$ & $0.96 \mathrm{M} / 4.74 \mathrm{M}$ & $0.44 \mathrm{M} / 0.42 \mathrm{M}$ & $139 / 415$ & $183 / 344$ \\
\hline Transitive & $19.7 \mathrm{M}$ & $1 \mathrm{~K}$ & $3.34 \mathrm{M} / 50.1 \mathrm{M}$ & $3.90 \mathrm{M} / 2.69 \mathrm{M}$ & $157 / 407$ & $834 / 1341$ \\
\hline CornerTurn & $18.1 \mathrm{M}$ & $22 \mathrm{~K}$ & $2.15 \mathrm{M} / 15.8 \mathrm{M}$ & $1.21 \mathrm{M} / 1.00 \mathrm{M}$ & $163 / 442$ & $1003 / 1434$ \\
\hline
\end{tabular}

of Impulse-MP. Larger problem sizes result in higher cache miss rates for the baseline version, so we believe that the benefit of Impulse-MP on real workloads will be no less than what is presented in this paper. In addition, the core loops of Matrix and Transitive are simulated only three times to save simulation time.

UVSIM models a micro-kernel that supports common system calls and realistic TLB miss handlers. All results presented in this paper come from complete simulations of the benchmark programs, including kernel and application time, and the direct and indirect overheads resulting from the use of remapping. However, initialization time is not included in the reported results to focus on sustained parallel performance. With real workloads, the majority of the total execution time is spent in the core loops, so the developers of the DIS Stressmarks encourage users not to include initialization time in reports.

4.3.1 Speedups. Our first set of experiments is done on a uniprocessor system and a 32-processor system. The resulting speedups of Impulse-MP over the baseline system are shown in Figure 5. In our study, the baseline system uses the write-invalidate protocol implemented in SN2-MIPS [SGI 2001]. The Impulse-MP system extends it with the Impulse MC and GET/PUT operations that are used solely for remapped data. Impulse-MP is very effective at improving the performance of these benchmarks. The speedups range from $3.4 \mathrm{X}$ to $10.8 \mathrm{X}$ with a geometric mean of $4.9 \mathrm{X}$ on a uniprocessor and from $4.9 \mathrm{X}$ to $24.4 \mathrm{X}$ with a geometric mean of $9.0 \mathrm{X}$ on a 32 -processor system.

Table III displays TLB and cache performance numbers on the 32-processor system. In this table, columns two and three present the number of TLB misses for the base and remapping-optimized versions. Columns four and five present the number of accesses that are satisfied by local or remote memory. Columns six and seven present the average latency of local and remote memory accesses measured in processor cycles. The large working sets and sparse access patterns in these benchmarks lead to significant numbers of TLB and cache misses in 


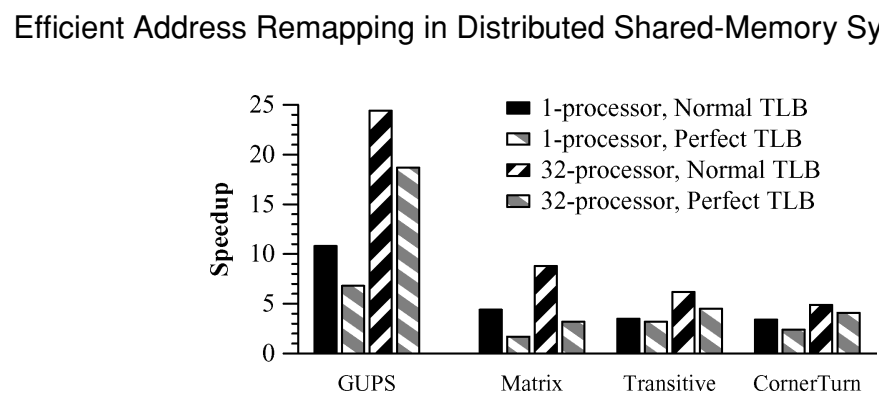

Fig. 6. Speedups of Impulse-MP, with perfect TLB.

the base system. Impulse-MP dramatically reduces the number of TLB and cache misses, eliminating about $99 \%$ of all TLB misses, $16 \%$ of local memory accesses, and $94 \%$ of remote memory accesses.

Gathering a cache line is much more expensive than fetching a single dense cache line. Cache lines composed of data that is gathered locally have an average latency of 537 cycles, versus an average of 169 cycles for nonremapped local accesses. Cache lines composed of remote data have an average latency of 950 cycles, while regular remote fetches have an average latency of only 436 cycles. However, the dramatic reduction in cache and TLB misses significantly overwhelms the increased memory latency and Impulse-MP delivers a significant performance improvement for every one of these benchmarks.

4.3.2 Effects of TLB. While it is hard to increase cache sizes to reduce cache misses, some systems use superpages to increase the effective TLB reach without increasing TLB size. To understand the effect of Impulse-MP on such systems, we simulate a perfect TLB that incurs only cold misses for the base system. Figure 6 shows the corresponding results. The average speedup of Impulse$\mathrm{MP}$ is reduced from $4.9 \mathrm{X}$ to $3.1 \mathrm{X}$ on the uniprocessor and from $9.0 \mathrm{X}$ to $5.8 \mathrm{X}$ on the 32-processor system. These results indicate that cache misses alone can slow down applications dramatically and that Impulse-MP can achieve significant performance gains even with the presence of superpages.

4.3.3 Effects of GET/PUT. Part of the benefit exhibited by Impulse-MP comes from extending the base coherence protocol to support fine-grained GET/PUT operations and release consistency. These same extensions could be made to the base system and exploited by the compiler/programmers without supporting Impulse-MP in its complete form. To isolate the effect of GET/PUT from the effect of gather-scatter, we extend our simulator to model a baseline system that supports GET/PUT and create versions of the benchmarks that exploit it by mapping sparsely-accessed data elements to a special uncacheable space. Accesses to this space bypass caches and result in byte-, word, or doubleword-grained GET/PUT operations, depending on the request size. We increase the number of MSHRs in this extended baseline model to match the number of GET/PUT requests supported by Impulse-MP, which results in a somewhat optimistic estimate of GET/PUT performance. Figure 7 presents the performance of Impulse-MP and the extended baseline system on these benchmarks. 


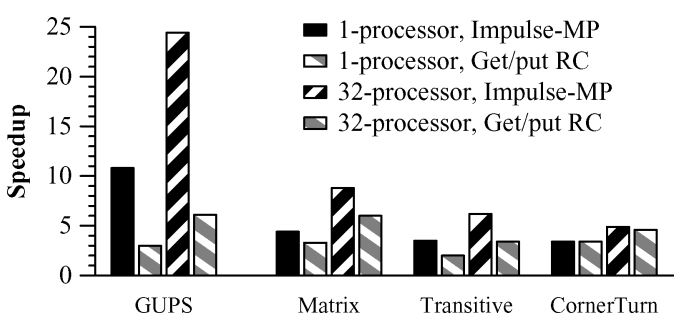

Fig. 7. Impulse-MP vs. GET/PUT.

Both GET/PUT and scatter/gather reduce the bandwidth requirements of an application. Scatter/gather also reduces latency by issuing a large number of memory requests in parallel, whereas the number of GET/PUTs operations that can be issued in parallel is limited by factors, such as instruction window size and load/store queue size. Thus, bandwidth-limited applications should benefit from both optimizations, while latency-limited applications should benefit more from scatter/gather.

Average memory latency is often a reflection of bandwidth limitations. As bandwidth becomes more and more of a bottleneck, effective memory latency increases. As Table III shows, GUPS and Matrix have low average memory latency, indicating that bandwidth is not the limiting factor in their performance. In contrast, Transitive and CornerTurn have high average memory latency, indicating that bandwidth is a limiting factor.

Consequently, as shown in Figure 7, Impulse-MP significantly outperforms GET/PUT for GUPS and Matrix, while Impulse-MP and GET/PUT perform similarly for CornerTurn. The only anomaly is that GET/PUT underperforms Impulse-MP on Transitive despite being a bandwidth-limited benchmark. The reason for this behavior is that Transitive accesses a single matrix in both column-major and row-major order simultaneously. Mapping the matrix to an uncacheable space so that column-major accesses are performed via GET/PUT operations, which is desirable due to their poor locality, also causes row-major accesses to be uncached, which hurts performance, since they could benefit from caching. On a system that supports coherent GET/PUT operations to cacheable data, we would expect GET/PUT to perform similarly to Impulse-MP on Transitive, but combining coherent GET/PUT operations and caching introduces nontrivial coherence problems.

4.3.4 Scalability. Figure 8 shows the scalability of the baseline and remapping-optimized versions of each program. The speedups in this figure are computed by comparing an Impulse-MP multiprocessor to an Impulse-MP uniprocessor and a base multiprocessor to a base uniprocessor. For each benchmark, the slope of the Impulse-MP is greater than the slope of the base system, showing that an Impulse-MP system scales better. For instance, the uniprocessor Impulse-MP system runs GUPS only 10.8 times faster than the uniprocessor base system, but the 32-processor Impulse-MP system runs GUPS 24.4 times faster than the 32-processor base system. The improved scalability is primarily due to Impulse's ability to vastly reduce the amount of coherence 

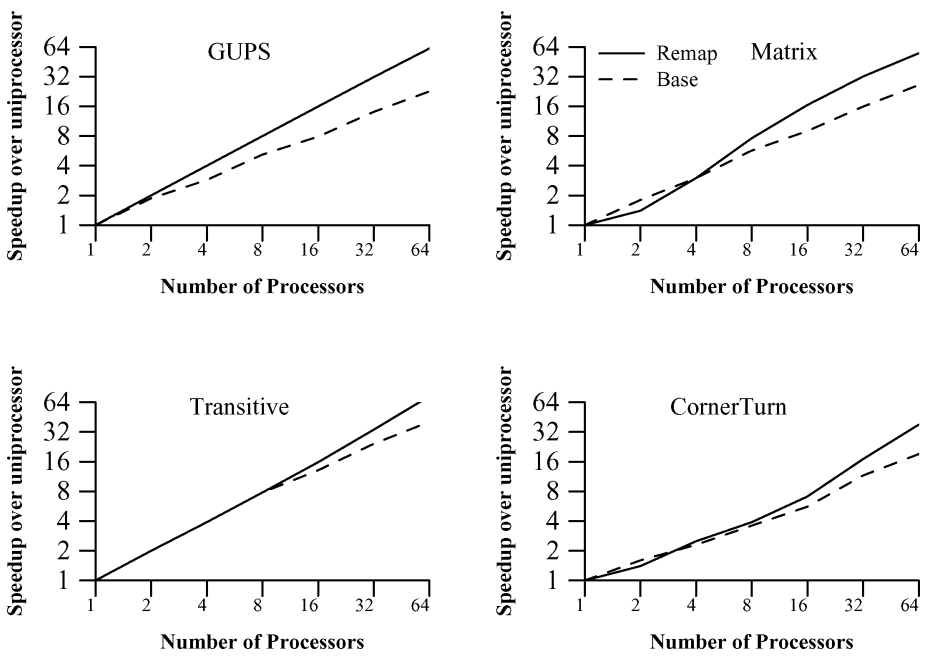

Fig. 8. Scalability of the base and Impulse-MP systems.

operations required to maintain coherence, which alleviates pressure on two bottlenecks to scalability: network bisection bandwidth and memory controller occupancy.

When going from a single processor to two processors, the base system scales better than Impulse-MP for all benchmarks. Our current implementation has two processors but only one shadow engine per node. The two processors compete for the same shadow engine, which reduces the benefit of Impulse remapping compared to the uniprocessor configuration. In the future we plan to investigate how many shadow engines should be instantiated per node to balance performance with cost.

4.3.5 Sensitivity to Memory / Network Latency. Figure 9 and 10 depicts how the performance of Impulse-MP fluctuates when memory and network latency are varied. The two figures show that that the speedup of Impulse-MP can change in either direction as the memory latency increases, but constantly increases as the network latency increases.

As memory latency increases, scatter/gather time increases. Because the Impulse memory controller performs sequential prefetching, the memory latency observed by a processor largely depends on whether or not a prefetch can complete before the corresponding demand request. For GUPS and Matrix, the processor takes a lot of time to consume a gathered line, which gives the memory controller sufficient time to complete a prefetch, effectively hiding the memory access latency from the processor. The processor-observed latency of shadow accesses for these two benchmarks is similar or even smaller than that of normal accesses, as shown in Table III. For these applications, increasing the memory latency increases the speedup of Impulse-MP, because the base runs now take longer but the Impulse-MP runtimes do not change much. For Transitive and CornerTurn, little computation is done on each line and the processor issues memory requests at a rate higher than the memory controller can handle. In 

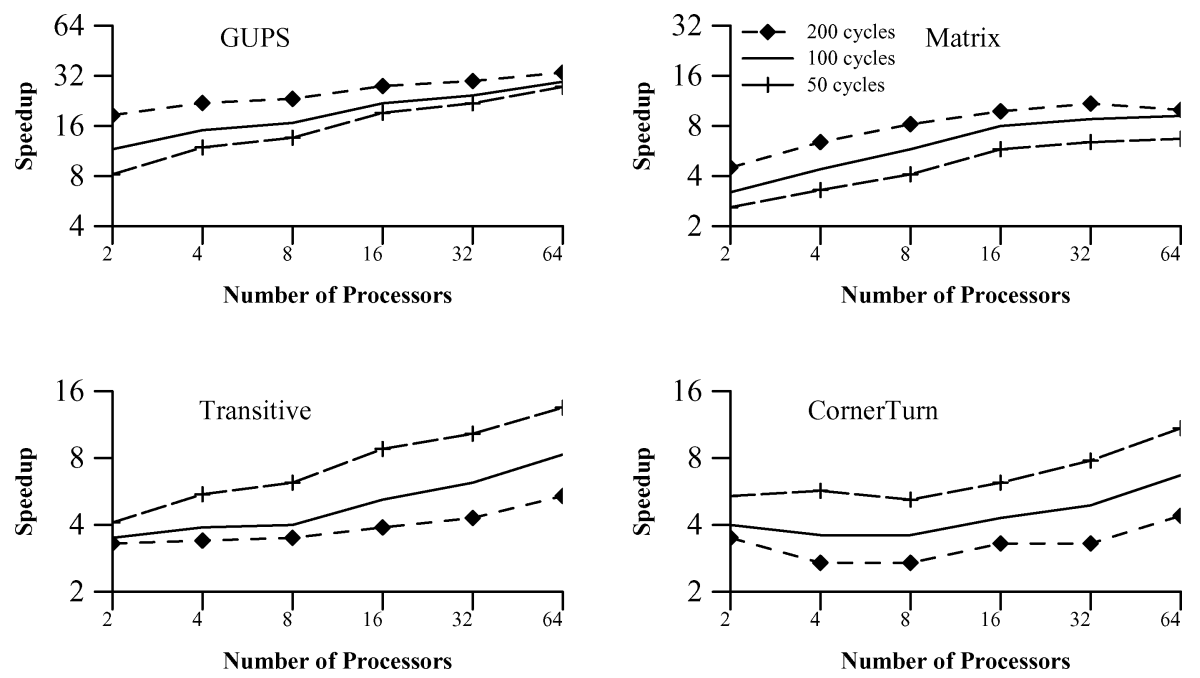

Fig. 9. Sensitivity to memory latency.

these cases, increasing memory latency reduces the speedup of Impulse-MP, because the processor observes much longer scatter/gather latency.

A major advantage of Impulse-MP is eliminating unnecessary network traffic and therefore significantly alleviating pressure on the network. In addition to the direct effects of eliminating remote memory accesses, eliminating coherence traffic results in fewer congestion-induced network delays. As network latency increases, the base system puts more pressure on the network and thus suffers more congestion, which, in turn, means that Impulse-MP may reduce more congestion-related delays. As a result, the benefit of Impulse-MP increases as the processor-network speed gap widens, as shown in Figure 10.

Figure 9 and 10 also show that the speedup of Impulse-MP generally increases as the number of processors increases. This is because of the better scalability of Impulse-MP, as explained in Section 4.3.4. The only anomaly is CornerTurn, for which Impulse-MP's speedup decreases going from four processors to eight processors. A closer look at the simulation results reveals that this effect is due to load imbalance-an artifact of the input and lazy page allocation policy we use. In this benchmark, each process handles an equal number of columns of the source matrix and the input we use has much better load balance with four processes than with eight processes. The ratio between the execution times of the longest process and shortest process is 1.10 on the four-processor system and 1.24 on the eight-processor system.

\section{RELATED WORK}

Our work is a direct extension to the Impulse system [Carter et al. 1999; Zhang et al. 2001]. We propose that the shadow engine be moved out of the memory controller to a location within the hub that can access remote memory. The shadow engine instead becomes an independent unit connected to the crossbar inside the hub so that remote requests generated by the shadow engine can be 

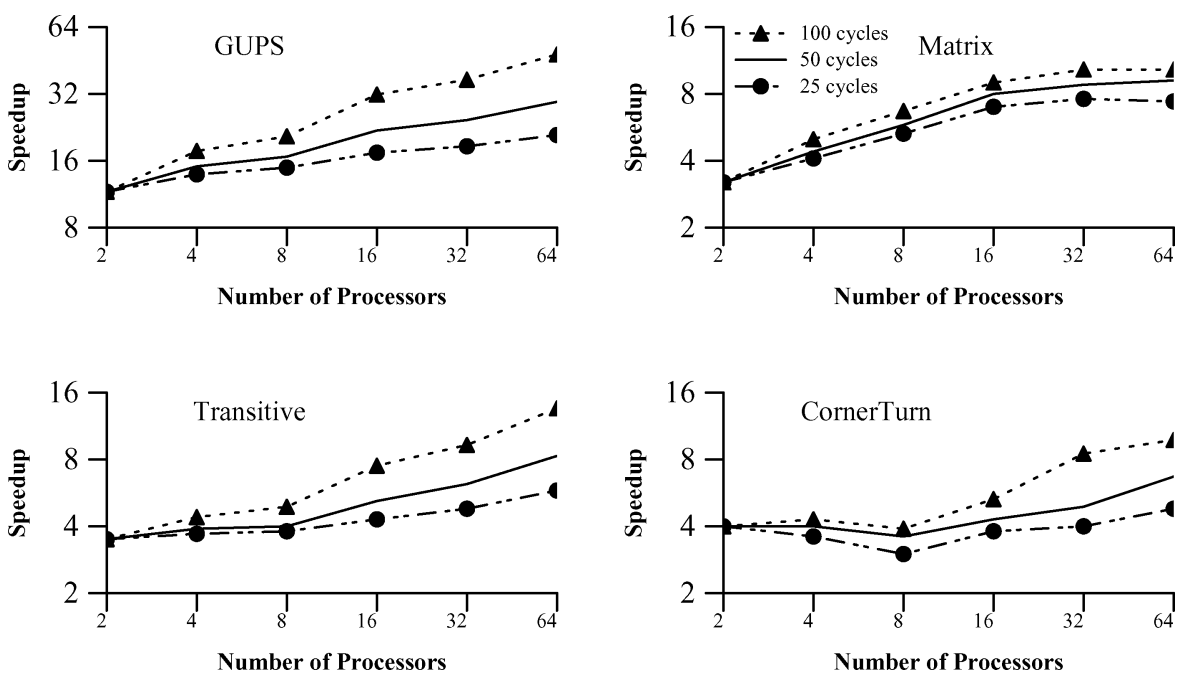

Fig. 10. Sensitivity to network latency.

easily routed to the home node. We also propose the use of fine-grained put and get operations to scatter and gather data from remote nodes.

The Active Memory architecture [Chaudhuri et al. 2002; Kim et al. 2003] implements similar functionality as Impulse-MP. It adds an extra bit (called the AM bit) to each directory entry to indicate whether or not any data in the line has been remapped and is being cached in some processor caches at a shadow address. When a shadow cache line is gathered, all lines in which data is fetched will have the AM bit set. When a normal access hits a line with the AM bit set, the directory controller uses another MTLB to find the shadow cache lines that contain data from this line. Active Memory achieves similarly impressive performance gains as Impulse-MP while ensuring full coherence for remapped data at the cost of extra hardware. Active Memory needs to support mapping from physical addresses to shadow address, in addition to shadow to physical mapping. To support indirect mapping, it must create the reverse vector of the indirection address vector. Active Memory works in coherent single-system images. By adopting a vectorlike PUT/GET model of coherence, ImpulseMP is easier to implement and thus we believe more likely to be adopted commercially.

The Cray T3E [Scott 1996] allows sparse data to be gathered using finegrained GETs and PUTs into contiguous E-registers that reside outside the processor core, substantially reducing the bus and network bandwidth that would have been required for normal cache line fills. Impulse-MP provides similar scatter/gather capabilities at the other side of the system bus, the Hub, without the need for special vector registers or a high-performance vector memory system. One key distinction between Impulse-MP and E-registers is that Impulse-MP can efficiently gather data distributed randomly (i.e., without a regular stride). The same functionality requires multiple E-register operations, one per gathered data item. Unlike Impulse-MP, the T3E does not use normal 
cache fill mechanisms, but instead issues GET/PUT requests from the processor, so the maximum sustainable bus bandwidth is reduced by about a factor of two.

In the same vein, Yamada [Yamada et al. 1994] has proposed instruction set changes to support combined relocation and prefetching into the L1 cache. Relocation is done at the processor in this system via regular cache fill requests and, thus, no bus and network bandwidth is saved.

Several researchers propose to incorporate processing power on DRAM chips, so-called PIM (processor-in-memory) systems [Hall et al. 1999; Oskin et al. 1998; Patterson et al. 1997; Rixner et al. 1998; Sunaga et al. 1996; Waingold 1997]. PIM architectures are adept at handling dense streaming or vector-style applications when all the required data resides within a single DRAM chip. However, once the data needed to perform a computation crosses a chip boundary, these systems effectively become a specialized form of distributed memory multiprocessors, with all of the attendant complexities. PIM architectures are useful in a variety of special-purpose domains, but much research remains to determine the extent to which they can be integrated into a general-purpose computing environment. The performance of these architectures is somewhat limited by the fact that DRAM processors are slower than logic processors and it is difficult to enforce data coherence in PIM architectures. In contrast to these projects, Impulse-MP does not make modifications to commodity DRAM chips. It merely needs a unit that can perform simple arithmetic operations and page table lookups and can be located on the memory controller and network interface die.

Adding intelligence to the memory controller is an approach taken by several researchers working to overcome the "Memory Wall" problem. The stream memory controller of McKee and et al. [1996] combines programmable stream buffers, prefetching within the memory controller, and intelligent DRAM scheduling. Corbal et al. [1998] propose a Command Vector Memory System that exploits parallelism and locality of reference to improve effective bandwidth for vector accesses on out-of-order vector processors. The SPARCstation20 [Donovan et al. 1995] uses a specialized pixel processor in its memory controller chip to accelerate certain graphics tasks. Solihin et al. [2002] propose adding a general-purpose processor core to a memory controller to direct prefetching into L2 cache. All of these projects have demonstrated that the potential benefits of using an advance memory system are significant. Impulse-MP attacks the network/memory latency problem from a different angle and is orthogonal to most of these approaches. For instance, it can be used with an intelligent DRAM scheduling to better schedule fine-grained DRAM accesses or it can be used with more advanced prefetching schemes to perform more accurate prefetching at the memory controller.

\section{CONCLUSIONS AND FUTURE WORK}

Two technology trends are causing severe performance bottlenecks for largescale multiprocessors: increasing memory latency and increasing network latency. The most evident manifestation of these trends is that the sustained 
performance of today's large-scale supercomputers for many data-intensive applications is less than 5\% of their peak performance [Graybill 2002].

The Impulse project attacks the memory bottleneck of single-node systems by adding a special form of "smarts" to the MC: application-specific physicaladdress remapping. Impulse allows applications to control how their data should be presented to processors at run time, thus greatly improving the efficiency of caches and TLBs and speeding up applications that do not perform well on conventional systems.

In this paper, we propose to implement these remapping mechanisms in a shared memory multiprocessor by moving the shadow engine out of the memory controller and by adding a few extensions to the coherence protocol to support fine-grained remote accesses. We demonstrate how several remapping functions improve the performance of four important data-intensive applications. On a 32-processor system, using indirect remapping improves the performance of GUPS and Matrix by factor of $24.4 \mathrm{X}$ and 8.8X, respectively. Using strided remapping improves the performance of Transitive and CornerTurn by factors of $6.2 \mathrm{X}$ and $4.9 \mathrm{X}$, respectively.

An important feature of Impulse-MP is that it modifies only the hub. No modifications to the CPU, caches, or DRAMs are necessary. As a result, we expect that it will be easier to get these ideas adopted into commercial systems than it will be for approaches that involve more radical hardware and programming model changes.

One of the remaining challenges is how to maintain coherence between two aliased views of remapped data. Thus, we have found little need for such hardware support, as treating remapped memory much like register data has worked well. However, we are considering mechanisms to track reverse mappings for remapped data. These mechanisms would allow a remote node coherently modify remapped data.

Remapping enables the processor TLB to use huge superpages in the shadow address space and moves fine-grained page translations to the MTLB. The MTLB works at the memory clock rate, is not in the processor's critical path, and is only accessed on cache misses, so it is possible to make it significantly larger and use a more sophisticated translation scheme than the processor TLB. We are also investigating alternate MTLB organizations that are capable of mapping petabyte ranges of memory in future large-scale supercomputers.

\section{REFERENCES}

AAE Corp. 2000. DIS Stressmark Suite. AAE Corp.

Carter, J., Hsieh, W., Stoller, L., Swanson, M., Zhang, L., Brunvand, E., Davis, A., Kuo, C.-C., Kuramkote, R., Parker, M., Schaelicke, L., and Tateyama, T. 1999. Impulse: Building a smarter memory controller. In Proceedings of the Fifth Annual Symposium on High Performance Computer Architecture. 70-79.

Chaudhuri, M., Kim, D., ANd HeinRICH, M. 2002. Cache coherence protocol design for active memory systems. In Proceeding of the International Conference on Parallel and Distributed Processing Techniques and Applications. 83-89.

Cheung, T. And Smith, J. 1986. A simulation study of the Cray X-MP memory system. IEEE Transactions on Computers C-35, 7 (July), 613-622. 
Corbal, J., Espasa, R., ANd VAlero, M. 1998. Command vector memory systems: High performance at low cost. In Proceedings of the 1998 International Conference on Parallel Architectures and Compilation Techniques. 68-77.

Cormen, T., Leiserson, C., AND Rivest, R. 1990. Introduction to Algorithms. McGraw Hill, New York.

Donovan, W., Sabella, P., Kabir, I., ANd Hsieh, M. 1995. Pixel processing in a memory controller. IEEE Graphics and Applications 15, 1 (Jan.), 51-61.

Fang, Z., Zhang, L., Carter, J., Hsieh, W., And McKee, S. 2001. Revisiting superpage promotion with hardware support. In Proceedings of the Seventh Annual Symposium on High Performance Computer Architecture. 63-72.

GrAYBILL, R. 2002. High productivity computing systems. http://www.darpa.mil/DARPATech2002/ presentations/ipto_pdf/speeches/GRAYBILL.pdf.

Hall, M., Kogge, P., Koller, J., Diniz, P., Chame, J., Draper, J., LaCoss, J., Granacki, J., Brockman, J., Srivastava, A., Athas, W., AND Freeh, V. 1999. Mapping irregular appilcations to DIVA, a PIM-based data-intensive architecture. In Supercomputing '99.

Huang, X., Wang, Z., AND McKinley, K. 2001. Compiling for an impulse memory controller. In Proceedings of the 2001 International Conference on Parallel Architectures and Compilation Techniques. 141-150.

Kim, D., Chaudhuri, M., ANd Heinrich, M. 2003. Active memory techniques for ccnuma multiprocessors. In Proceedings of International Symposium on Parallel and Distributed Systems.

Lenoski, D., Laudon, J., Gharachorloo, K., Gupta, A., And Hennessy, J. 1990 . The directory-based cache coherence protocol for the DASH multiprocessor. In Proceedings of the 17th Annual International Symposium on Computer Architecture. 148-159.

LOVETT, T. AND CLAPP, R. 1996. STiNG: A CC-NUMA compute system for the commercial marketplace. In Proceedings of the 23rd Annual International Symposium on Computer Architecture. 308-317.

McKeE, S. ET AL. 1996. Design and evaluation of dynamic access ordering hardware. In Proceedings of the 1996 International Conference on Supercomputing. 125-132.

MIPS 2002. MIPS R18000 Microprocessor User's Manual, Version 2.0. MIPS.

MooRe, G. 1965. Moore's law. http://www.intel.com/research/silicon/mooreslaw.htm.

Oskin, M., Chong, F., AND Sherwood, T. 1998. Active pages: A model of computation for intelligent memory. In Proceedings of the 25th Annual International Symposium on Computer Architecture. 192-203.

Patterson, D., Anderson, T., Cardwell, N., Fromm, R., Keaton, K., Kozyrakis, C., Thomas, R., and Yelick, K. 1997. A case for Intelligent RAM: IRAM. IEEE Micro 17, 2 (Apr.).

Rixner, S., Dally, W., Kapasi, U., Khailany, B., Lopez-Lagunas, A., Mattson, P., and Owens, D. 1998. A bandwidth-efficient architecture for media processing. In Proceedings of IEEE/ACM 31st International Symposium on Microarchitecture.

Sсотт, S. 1996. Synchronization and communication in the T3E multiprocessor. In Proceedings of the 7th Symposium on Architectural Support for Programming Languages and Operating Systems.

SGI. 2001. SN2-MIPS Communication Protocol Specification, Revision 0.12. SGI.

SGI. 2002. Orbit Functional Specification, Vol. 1, Revision 0.1. SGI.

Solinin, Y., LeE, J., AND ToRRELlas, J. 2002. Using a user-level memory thread for correlation prefetching. In Proceedings of the 29th Annual International Symposium on Computer Architecture. 171-182.

Sunaga, T., Kogge, P. M. ET AL. 1996. A processor in memory chip for massively parallel embedded applicatiions. IEEE Journal of Solid State Circuits, 1556-1559.

Swanson, M., Stoller, L., AND CARTER, J. 1998. Increasing TLB reach using superpages backed by shadow memory. In Proceedings of the 25th Annual International Symposium on Computer Architecture. 204-213.

WaINGOLD, E. ET AL. 1997. Baring it all to software: Raw machines. IEEE Computer, 86-93.

Yamada, Y., GyllenhaAl, J., HaAB, G., AND HwU, W.-M. 1994. Data relocation and prefetching for programs with large data sets. In Proceedings of IEEE / ACM 27th International Symposium on Microarchitecture. 118-127.

ZHANG, L. 2000. URSIM reference manual. Tech. Rep. UUCS-00-015, University of Utah. August. 
Zhang, L., Pingali, V. K., Chandramouli, B., and Carter, J. 2000. Memory system support for dynamic cache line assembly. In The Second Workshop on Intelligent Memory Systems.

Zhang, L., Fang, Z., Parker, M., Mathew, B., Schaelicke, L., Carter, J., Hsieh, W., and McKee, S. 2001. The Impulse memory controller. IEEE Transactions on Computers 50, 11 (Nov.), 11171132.

Received March 2005; revised June 2005; accepted February 2006 